\title{
Role of Sepsis Screen Parameters in Early Diagnosis of Neonatal Septicemia
}

\author{
Lazarus Monica, J. Seth Riti* and B. Kinnare Amit \\ Department of Pediatrics \& Department of Microbiology, NSCB Medical College, \\ Jabalpur, India \\ *Corresponding author
}

\begin{tabular}{|l|}
\hline Ke y w o r d s \\
Blood culture, C- \\
reactive protein, \\
Neonatal septicemia, \\
Sepsis screen
\end{tabular}

A B S T R A C T

Neonatal sepsis is a clinical syndrome of bacteremia characterized by systemic signs and symptoms of infection in the first month of life. It has been a challenge with its high incidence and grave prognosis, despite adequate treatment with modern antibiotics. The signs and symptoms are protean with a high mortality and thus there is urgent need to know whether the baby has sepsis. Confirmation of the diagnosis by definitive culture is not rapidly possible. Sepsis screen tests involving WBC indices and CRP form simple, cheap, rapid, early and easily available parameters and these can be used as non-specific indicators of bacterial sepsis. Thus this study was conducted to determine the Sensitivity, Specificity, Predictive values of sepsis screen tests, as an indicator of neonatal sepsis in comparison with blood culture. In this prospective study, blood samples were collected from clinically suspected cases of neonatal sepsis and were Investigated for estimation of C-reactive protein, Total WBC count, Absolute neutrophil count, Band cell count and I/T ratio. Blood Culture and Antibiotic susceptibility testing were performed as per standard protocols. Out of 80 cases studied, 41 cases $(51.25 \%$.) yielded a positive culture. I/T ratio and CRP have good sensitivity and negative predictive value. Neutropenia and leucopenia have highest specificity and positive predictive value. If $>2$ of the above tests are positive, sensitivity and negative predictive value of the screening tool increased to more than $90 \%$. Thus sepsis screening parameters are easily available, rapid, cost effective screening tests with good sensitivity and negative predictive value so that if any of the two screening tests are negative, the infection is unlikely. CRP alone is also a good diagnostic and therapeutic tool. It has high sensitivity and a good negative predictive value.

\section{Introduction}

Neonatal sepsis is a clinical syndrome of bacteremia characterized by systemic signs and symptoms of infection in the first month of life. It encompasses systemic infection of the newborn including septicemia, meningitis, pneumonia, arthritis, osteomyelitis and urinary tract infection of the newborn. Globally of the
130 million babies born every year, about 4 million die in the first 4 weeks of life, i.e. the neonatal period. The main direct causes of neonatal deaths are estimated to be preterm birth (28\%), severe infection (26\%), and birth asphyxia (23\%). It is estimated that up to $20 \%$ of neonates develop sepsis and approximately $1 \%$ die of sepsis-related causes (Khatua et al., 1986). Neonatal survivors of sepsis can have 
severe neurological sequelae due to CNS infections, as well as from secondary hypoxemia resulting from septic shock persistent pulmonary hypertension and severe parenchymal lung disease.

Neonatal septicemia with its high incidence and grave prognosis, despite adequate treatment with modern antibiotics, has been a challenge all times. Optimal diagnosis and treatment strategies are difficult to define. The signs and symptoms are protean with a high mortality and thus there is urgent need to know whether the baby has sepsis, to institute treatment as quickly as possible. Confirmation of the diagnosis by definitive culture is not rapidly possible.

The concentration of many serum proteins rises in response to inflammation, associated with infection, trauma or tissue damage. Amongst these proteins, the important ones are CRP, haptoglobin and fibrinogen. These can be used as non-specific indicators of bacterial sepsis. Sepsis screen tests involving WBC indices and CRP form simple, cheap, rapid, early and easily available parameters with reasonable diagnostic accuracy especially when they are used in combination. On this basis, early and rational antibiotics therapy can be started in critical septicemic infants. Sepsis related mortality is largely preventable with rational antimicrobial therapy and aggressive supportive care.

The main aim of this study to determine the Sensitivity, Specificity, Predictive values of sepsis screen tests, as an indicator of neonatal sepsis in comparison with blood culture.

\section{Materials and Methods}

This prospective study was conducted in the Department of Paediatrics, NICU and Department of Microbiology at this Medical College and Hospital, from September 2010 to
August 2011. The blood samples were collected from 148 clinically suspected cases of neonatal sepsis which were sent to the pathology and microbiology department of the same college for evaluation. But of these all the required investigations of only 80 patients could be traced. The remaining 68 had to be excluded on account of some missing investigations or due to non- compliance of relatives and other inevitable circumstances like dropouts. Detailed history and clinical findings were recorded. Study was approved by institutional ethics committee.

\section{Inclusion criteria}

Neonates presenting with Perinatal or clinical risk factors like Low birth weight, Prematurity, Birth asphyxia, Home delivery, History of PROM in mother for more than 24 hours, Maternal fever, Instrumentation, Poor feeding, lethargy, reduced activity, Sclerema, Hypothermia/fever, Jaundice, Apnea, tachypnea, Abdominal distension and vomiting, Diarrhea, Skin mottling, Bleeding tendencies, Seizures were enrolled for the study. However Neonates who received antibiotics before admission or having major congenital malformations were excluded.

All neonates were categorized into early onset sepsis EOS (0-72 hours) or late onset sepsis LOS ( $>3$ days) sepsis based on the day of presentation in the postnatal life. Detailed history and clinical findings were recorded as per the proforma. Among early onset sepsis, perinatal risk factors were noted and each baby was given a score (Barbara et al., 1979; Tallur et al., 2000) based on these risk factors(given in parenthesis for each factor) i.e. Foul smelling liquor (2), Unclean vaginal examination done before delivery (2), Duration of labour exceeding 24 hours (2), Birth asphyxia (Apgar < 6 at $1 \mathrm{~min}$ ) (2), Birth weight $2.5 \mathrm{Kgs}$ or less and/ or gestation age $<37$ weeks (3), Duration of rupture of 
membrane before delivery $>24$ hours (1), and Maternal pyrexia (1) thus making a total score of 13.

Babies with Score 0-3 were observed clinically while with Score $>4$ were Investigated. All neonates who were clinically symptomatic or having a septic score $>4$, their blood sample was collected taking aseptic precautions, out of which $2 \mathrm{ml}$ of the blood sample was inoculated aseptically into a blood culture bottle containing brain heart infusion broth, $1 \mathrm{ml}$ of the blood was allowed to clot in a sterile plain vial to collect serum for estimation of $\mathrm{C}$-reactive protein and the remaining $1 \mathrm{ml}$ was collected in a vial containing the anticoagulant EDTA for estimation of the Total WBC count, Absolute neutrophil count, Band cell count and I/T ratio. Relevant to the clinical situation CSF, urine analysis and swabs from infective focus were taken. Blood Culture was performed as per standard protocols. Antibiotic susceptibility testing was done for all the isolates on Mueller Hinton agar using commercially available discs (himedia), by Kirby Bauer disc diffusion technique as per the CLSI guidelines (CLSI, 2010). CRP test was done by using diagnostic kit for in-vitro detection of CRP in human serum by the rapid slide latex agglutination qualitative method using a commercial kit as per manufacturer's instructions and a CRP value of $>0.6 \mathrm{mg} / \mathrm{dl}$ was taken as a positive test.

The Total leucocyte count, differential count, Absolute Neutrophil Count, Band cell count, $\mathrm{I} / \mathrm{T}$ ratio and platelet count were calculated using the automated cell counter for better results and quality control. Hemoglobin was estimated using the Sahli's method.

The cut off values of the positive rapid screening tests in this study were C-Reactive protein (CRP): > 0.6mg/dl, Total leucocyte count (Leukopenia): < 5,000 cells/cu.mm,
Absolute neutrophil count (Neutropenia): < 1,750 cells/cu.mm, Band cell count to total neutrophil count ratio (I/T): $>0.2$, Platelet count (thrombocytopenia): < 1.5 lakhs/cu.mm.

If CRP was positive, the empirical antibiotic therapy was started according to antibiotic guidelines in the NICU, awaiting the culture reports. The duration of treatment and hospital stay was noted in all neonates. The discharged neonates were followed up at NICU/ Paediatric OPD.

The statistical analysis was done using the results of the present study. Sepsis score and sepsis screen test results were compared with the blood culture results as the gold standard. Data analysis was carried out using Statistical Package for Social Sciences for windows (SPSS Inc 1999, New York, version 11) package.

\section{Results and Discussion}

The results from 80 patients, whose follow-up could be completed, were considered for analysis. Out of 80 cases studied, 41 cases yielded a positive culture, giving a positivity rate of $51.25 \%$. Higher proportion of late onset sepsis $(66.66 \%)$ were culture positive as compared to early onset sepsis (44.66\%), which was not statistically significant.

Comparing the affection rates, the male $(67.5 \%)$ to female $(32.5 \%)$ ratio was approximately $2: 1$. But this is not significant with respect to the rates of culture positivity. 46 cases $(57.5 \%)$ were preterm, of which 27 $(65.8 \%)$ were culture positive. The distribution of cases as EOS and LOS were not statistically significant with respect to the gestational age. However, 58 (72.5\%) cases were $<2.5 \mathrm{~kg}$ and $14(17.5 \%)$ of them being VLBW (1-1.5 kg). Amongst the 41 culture positives, $31(75 \%)$ were $<2.5 \mathrm{~kg}$ and it was significant. ( $\square$ value is 4.04 : p value $<0.05$ ). 
19 of $25(76 \%)$ of the early onset culture proven sepsis had their birth weight $<2.5 \mathrm{~kg}$. Amongst LOS also, $76 \%$ of them were $<2.5 \mathrm{~kg}$. $78 \%$ of the spontaneously delivered infants were positive for culture. $22 \%$ of the culture positives were assisted deliveries.

Out of the 56 EOS babies, higher proportion were preterm and had birth asphyxia as a risk factor both in culture proven and culture negative cases. So no significance was found between the 2 groups. Higher proportion of culture proven sepsis had intra partum risk factors like Labour duration >24hours (mostly outside/ emergency cases), Unclean/ repeated vaginal examinations, Foul smelling liquor and they were significantly associated with culture proven sepsis. PROM> 24 hours and maternal fever had no significant association. Higher proportion of babies with culture proven sepsis had $>2$ risk factors.

Out of the 24 cases of LOS, Higher proportion of LOS babies were preterm and LBW and had previous admission to the hospital in prior 1 week as a risk factor. More often they were due to faulty feeding practices, prelacteal feeds and some focus of infection as risk factors for sepsis. Predominant clinical findings in these patients were Refusal of feeds (72.5\%), Lethargy and weak reflexes (81.5\%), Hypothermia (26.2\%), Hyperthermia (13.75\%), Cyanosis (22.5\%), Dehydration (6.25\%), Tachypnoea (37.5\%), Apnea (16.25\%), Chest retraction (31.25\%), Tachycardia (48.75\%), and Shock (CRT>3 sec), Loose stools, Abdominal distension, Jaundice, Vomiting, High pitched cry, Convulsion, Bulging AF, Bleeding manifestation, Sclerema in varying proportions. More than one finding could be seen in one patient. Higher proportion of septic babies had vague symptoms like variation in established feeding pattern with failure to suck and lethargy. Lethargy is also a danger sign of returning back immediately in the hospital, which was explained to all the mothers whose babies were discharged from the hospital and hence was an important sign with babies with LOS.

$\mathrm{I} / \mathrm{T}$ ratio and $\mathrm{CRP}$ were positive in higher proportion of culture positive cases. Neutropenia, leucopenia and thrombocytopenia were positive in higher proportions in culture proven cases compared to culture negative cases. Many of the screening parameters are significant with respect to culture proven sepsis.

I/T ratio and CRP have good sensitivity and negative predictive value. Neutropenia and leucopenia have highest specificity and positive predictive value. If $>2$ of the above tests are positive, sensitivity and negative predictive value of the screening tool increased to more than $90 \%$.

Septicemia was the most common cause of clinical presentation, constituting about $48.75 \%$ of cases, followed by pneumonia and meningitis. Among early onset sepsis, septicemia $(60.3 \%)$ followed by pneumonia $(36 \%)$ was the most common presentation. Among LOS, pneumonia (37.5\%) followed by meningitis $(25 \%)$ and infective diarrhea were the most common modes of presentation.

Out of 41 culture positive cases, 14 (34.14\%) were gram positive and $27(65.85 \%)$ were gram negative isolates. Klebsiella (46.3\%), followed by $S$. aureus (29.2\%) was the most common isolates.

Overall mortality rate in the 80 cases studied was $33.75 \%$. There was no statistical difference among the early and the late onset cases. CFR was higher in the culture positive group $(43.9 \%)$ as compared to the culture negative group (23\%) and this was statistically significant $\left(\square^{2}=3.88, \mathrm{p}<0.05\right)$. No statistical significance was attributed to the differences 
seen in distribution with respect to place of delivery or sex of the neonate. Death rates were higher in LBW/VLBW babies (24 out of 58 i.e. $41.38 \%$ ) as compared with normal birth weight babies ( 3 out of 22 i.e. $5.17 \%$ ) and it was statistically significant $\left(\square^{2}=4.10\right.$, $\mathrm{p}<0.05$ ). Total case fatality was more amongst the preterm babies i.e. $77.7 \%$ and it is statistically significant. $\left(\square^{2}=4.96, p<0.05\right)$. CFR among culture positive preterm babies was $44.68 \%$, while it was $18.18 \%$ among term babies which was again statistically significant. $\left(\square^{2}=4.18, \mathrm{p}<0.05\right)$. Neutropenia and thrombocytopenia are good predictors of septicemia and mortality.

Sepsis with MODS was the most common causes of death in clinically suspected group, while sepsis with pneumonia accounted for the next commonest cause. Case fatality was highest due to sepsis with gram negative organisms, Klebsiella being the most common and alone contributing to $54.4 \%$ of mortality.

In this study, our basic aim was to study neonates with suspected septicemia and correlate significance of various sepsis screen parameters which are possible at our set-up to catch them early and institute appropriate treatment, to ensure better survival and prevent morbidity.

It was seen that many neonates who were enrolled in the study, could be easily excluded by simple measures like proper positioning of the baby during breast feed (for those complaining of feed refusal), Kangaroo mother care (for those with complaints of hypothermia). Also many babies were excluded, as they did better by thorough counselling and explanation of danger signs as per IMNCI guidelines of the UNICEF.

In the present study, an attempt has been made to correlate the efficacy of the Sepsis score and the Sepsis screen parameters with the blood culture results. Maximum culture positive cases were seen in neonates less than 3 days old with early onset septicemia.

Similar observations were seen in previous studies (Khatua et al., 1986; National Neonatal Perinatal Database 2002-03; Stoll et al., 1996) which also reported a higher proportion of early onset septicemia cases. EOS could be due to ascending infection following rupture of membranes or during trial of labour such as at home or in PHCs/ CHCs. Many of these cases are referred to the Medical College from far off destination on an emergency basis.

The higher proportion of EOS cases may be due to the immature immunological responses of the neonates exposed to risk factors in the first week of life, making them more susceptible to infection in this period.(Stoll et al., 1996).

The proportion of culture positive septicemia cases was higher among the preterm neonates in the present study, which is comparable to past Indian studies (Joseph et al., 1984; Joshi et al., 2000; Philip and Mills, 2000). According to (Barbara et al., 1979) and (Stoll el al., 1996), the incidence of septicemia is inversely proportional to gestational age of the neonates which is similar in this study. In the present study, approximately $50 \%$ of the culture positive cases were $<2.5 \mathrm{~kg}$, which is comparable to studies done by other authors.

Present study shows higher proportion of culture positive cases in inborn admission, which is comparable with other studies. This probably reflects that hospital, being a tertiary referral hospital for both Obstetrics and Pediatrics cases, has maximum late referral and intervened cases with higher proportion of babies born with adverse intrapartum risk factors for neonatal sepsis (Table 1-3). 
Table.1 Correlation of sepsis screen parameters with the blood culture status

\begin{tabular}{|c|c|c|c|c|c|}
\hline $\begin{array}{l}\text { Sl. } \\
\text { No }\end{array}$ & $\begin{array}{l}\text { Screening } \\
\text { parameters }\end{array}$ & $\begin{array}{l}\text { Culture } \\
\text { Positives } \\
\mathbf{n}=\mathbf{4 1}(\%)\end{array}$ & $\begin{array}{l}\text { Culture } \\
\text { negative } \\
\mathrm{n}=39(\%)\end{array}$ & $\begin{array}{l}\text { Total cases } \\
n=80\end{array}$ & $\begin{array}{l}\square^{2} \\
\text { (p value) }\end{array}$ \\
\hline 1. & $\begin{array}{l}\text { CRP positive } \\
(>0.6 \mathrm{mg} / \mathrm{dl})\end{array}$ & $32(78 \%)$ & $23(58.9)$ & $55(68.75 \%)$ & $\begin{array}{l}3.38 \\
(>0.05)\end{array}$ \\
\hline 2. & $\begin{array}{l}\text { Leucopenia } \\
(<5000 / \mathrm{cmm})\end{array}$ & $\begin{array}{l}12 \\
(29.2 \%)\end{array}$ & $2(5.1)$ & $14(17.5 \%)$ & $\begin{array}{l}8.07 \\
(<0.01)\end{array}$ \\
\hline 3. & $\begin{array}{l}\text { Neutropenia } \\
(<1750 / \mathrm{cmm})\end{array}$ & $7(17 \%)$ & $1(2.56)$ & $\begin{array}{l}8 \\
(10 \%)\end{array}$ & $4.68(<0.05)$ \\
\hline 4. & $\begin{array}{l}\text { Thrombocytopenia } \\
\text { (<1.5 lakh/cmm) }\end{array}$ & $11(26.8 \%)$ & $3(7.69)$ & $\begin{array}{l}14 \\
(17.5 \%)\end{array}$ & $5.07(<0.05)$ \\
\hline 5. & $\mathrm{I} / \mathrm{T}$ ratio $>0.2$ & $33(80.4 \%)$ & $22(56.4)$ & $55(68.75 \%)$ & $\begin{array}{l}5.39 \\
(<0.05)\end{array}$ \\
\hline 6. & $\begin{array}{l}\text { Any two or more tests } \\
\text { positive }\end{array}$ & $40(97.5 \%)$ & $15(38.46)$ & $55(68.75 \%)$ & $32.49(<0.0001)$ \\
\hline
\end{tabular}

Table.2 The sensitivity, specificity, positive predictive accuracy and negative predictive accuracy of sepsis screen parameters (Considering blood culture as the gold standard)

\begin{tabular}{|l|l|l|l|l|l|}
\hline $\begin{array}{l}\text { Sl. } \\
\text { No }\end{array}$ & $\begin{array}{l}\text { Sensitivity } \\
(\%)\end{array}$ & $\begin{array}{l}\text { Specificity } \\
(\%)\end{array}$ & $\begin{array}{l}\text { PPV } \\
(\%)\end{array}$ & $\begin{array}{l}\text { NPV } \\
(\%)\end{array}$ \\
\hline 1. & $\begin{array}{l}\text { CRP } \\
(>0.6 \mathrm{mg} / \mathrm{dl}) \\
(>0.6 \mathrm{mg} / \mathrm{dl})\end{array}$ & 78 & 41 & 58.18 & 64 \\
\hline 2. & $\begin{array}{l}\text { Leucopenia } \\
(<5000 / \mathrm{cmm})\end{array}$ & 31.70 & 94.8 & 85.7 & 56 \\
\hline 3. & $\begin{array}{l}\text { Neutropenia } \\
(<1750 / \mathrm{cmm})\end{array}$ & 17 & 97.4 & 87.5 & 57.5 \\
\hline 4. & $\begin{array}{l}\text { Thrombocytopenia } \\
(<1.5 \text { lakh/cmm })\end{array}$ & 26.8 & 92.3 & 78.5 & 54.54 \\
\hline 5. & $\begin{array}{l}\text { I/T ratio }>0.2 \\
\text { 6. }\end{array}$ & 80.48 & 43.58 & 60 & 68 \\
\hline Two or more tests positive & 97.5 & 61.5 & 72.5 & 96 \\
\hline
\end{tabular}


Table.3 Comparison of sensitivity, specificity positive predictive accuracy and negative predictive accuracy of sepsis screen parameters in various studies (Anita Chandna et al., 1988; Elizabeth Mathai et al., 2004; Gerdes, 2004; National Neonatal Perinatal Database, 2002-03; Ronald Misquith et al., 2004; Sharmila Ghosh et al., 2001; Ved Parkash Takkar et al., 1974)

(Considering blood culture as the gold standard)

\begin{tabular}{|c|c|c|c|c|c|c|c|c|c|}
\hline \multirow[t]{2}{*}{$\begin{array}{l}\text { Sl. } \\
\text { No }\end{array}$} & \multirow[t]{2}{*}{$\begin{array}{l}\text { Screening } \\
\text { parameters }\end{array}$} & \multicolumn{2}{|c|}{$\begin{array}{c}\text { Sensitivity } \\
(\%)\end{array}$} & \multicolumn{2}{|c|}{$\begin{array}{c}\text { Specificity } \\
(\%)\end{array}$} & \multicolumn{2}{|c|}{$\begin{array}{r}\text { PPV } \\
(\%)\end{array}$} & \multicolumn{2}{|c|}{$\begin{array}{r}\text { NPV } \\
(\%)\end{array}$} \\
\hline & & $\begin{array}{l}\text { Previous } \\
\text { studies }\end{array}$ & $\begin{array}{l}\text { Present } \\
\text { study }\end{array}$ & $\begin{array}{l}\text { Previous } \\
\text { studies }\end{array}$ & $\begin{array}{l}\text { Present } \\
\text { study }\end{array}$ & $\begin{array}{l}\text { Previous } \\
\text { studies }\end{array}$ & $\begin{array}{l}\text { Present } \\
\text { study }\end{array}$ & $\begin{array}{l}\text { Previous } \\
\text { studies }\end{array}$ & $\begin{array}{l}\text { Present } \\
\text { study }\end{array}$ \\
\hline 1. & $\begin{array}{l}\text { CRP } \\
(>0.6 \mathrm{mg} / \mathrm{dl})\end{array}$ & $52-93$ & 78 & $60-94$ & 41 & $7-91$ & 58.18 & $14-99$ & 64 \\
\hline 2. & $\begin{array}{l}\text { Leucopenia } \\
(<5000 / \mathrm{cmm})\end{array}$ & $22-100$ & 31.7 & $51-89$ & 94.8 & $12-51$ & 85.7 & $35-100$ & 56 \\
\hline 3. & $\begin{array}{l}\text { Neutropenia } \\
(>1750 / \mathrm{cmm})\end{array}$ & $38-77$ & 17 & $49-92$ & 97.4 & $35-90$ & 87.5 & $69-99$ & 57.5 \\
\hline 4. & $\begin{array}{l}\text { Thrombocytopenia } \\
(<1.5 \mathrm{lkh} / \mathrm{cmm})\end{array}$ & $22-57$ & 26.8 & $53-100$ & 92.3 & $39.7-100$ & 78.5 & $70-94$ & 54.54 \\
\hline 5. & $\mathrm{I} / \mathrm{T}$ ratio $>0.2$ & $52-100$ & 80.4 & $30-95$ & 43.5 & $11-92$ & 60 & $22-100$ & 68 \\
\hline 6. & $\begin{array}{l}\text { Two or more tests } \\
\text { positive }\end{array}$ & $53-100$ & 97.5 & $23-92$ & 61.5 & $19-51$ & 72.5 & $98-100$ & 96 \\
\hline
\end{tabular}

The culture positive neonatal septicemia cases were higher among the males than the females, showing a ratio of 1.4: 1 and these results are comparable with the observations made by others authors. The male preponderance in neonatal septicemia may be linked to the $\mathrm{X}$ - linked immune no regulatory gene resulting in the host's susceptibility to infections in the males. In the present study single CRP value has sensitivity of $78 \%$ and Negative predictive value of $64 \%$ which is comparable to observation made by other studies. The difference in various studies is due to different cut-off value used in the qualitative test (kit). We had a higher sensitivity because our cut-off was $0.6 \mathrm{mg} / \mathrm{dl}$.

Leucopenia i.e. total WBC counts $<5000$ cells/ cu.mm is one of the diagnostic criteria for detecting neonatal septicemia. In our study, leucopenia has high specificity and positive predictive value. This is comparable with results quoted by Gerdes (2004). The differences in the results of this parameter shown by the different studies(sensitivity 22$100 \%$ and specificity $51-94.58 \%$ ) may be due to variations in the blood sampling time, the severity of infection, the age of the neonates, and the reduced sensitivity of this test in the first week of life. Neutropenia (absolute neutrophil count $<1750$ cells/ cumm) had a high specificity but low sensitivity in present study which is comparable with the past observations (Gerdes, 2004; Sharmila Ghosh et al., 2001; Varsha et al., 2003). I/T ratio has a high sensitivity and a negative predictive value; low specificity and positive predictive value, which is comparable to the observation made by Gerdes (2004) however thrombocytopenia has high specificity and 
positive predictive value as sepsis screening tool which is comparable with observation made by Sharmila Ghosh et al., (2001) and Ronald Misquith et al., (2004).

In the present study, the differences in the results of this parameter shown by the different studies may be due to the variations in the blood sampling time, the severity of infection, the age of the neonates, the diagnostic criteria followed and the reduced sensitivity of this test after the first week of life.

Considering any combination of two tests (one of the haematological parameters and CRP) being positive as a screening tool for sepsis, the sensitivity and negative predictive value increased but specificity and positive predictive value remained the same in our study, this being comparable to the observations in past studies (Anita Chandna et al., 1988; Gerdes, 2004; National Neonatal Perinatal Database, 2002-03).

In the present study, 41 of the 80 cases studied were culture positive, giving a positivity rate of $51.25 \%$. Which were comparable with the studies conducted by Tallur et al., (2000), Roy et al., (2002) and Rekha Sriram (2011), while studies conducted by Joshi et al., (2000), Madhu Sharma et al., (2002) and NNPD (National Neonatal Perinatal Database, 2002-03) showed a very low culture positivity. The culture positivity depends on time of sampling, extent of bacteremia in neonate and prior antibiotic treatment in the neonate.

We had Klebsiella pneumoniae (46.3\%) was the predominate isolate, followed by $S$. aureus (29.2\%). Gram negative organisms formed the majority of the isolates as compared to Gram positive organisms (65.8\% vs. $34.1 \%$ respectively) in the present study, comparable with studies conducted by others.
Higher proportion of mortality was associated with early onset culture proven sepsis and most of them were preterm and or low birth weight. This was statistically significant ( $p$ value $<0.05$ ) in the present study which is comparable with studies of other authors.

Neutropenia and thrombocytopenia are good predictors of mortality with about $80-90 \%$ cases suffering fatality, which is comparable to other studies. Mortality rate was high among culture positive cases (isolates mostly being gram negative organisms). Gram negative sepsis is associated with high mortality. This is probably related to lack of specific IgM antibodies and complement deficiency in newborn which are required for protection against gram negative organism.

In the present study, there was high case fatality rate among babies presenting with difficulty in breathing, hypothermia, blood in stools, abdominal distension, lethargy/excessive irritability or refusal of feeds and those who developed refractory shock, DIC, respiratory failure, meningitis and NEC during the course of illness and it was comparable with other studies.

This probably reflects that higher proportion of babies had infection with highly virulent gram negative organisms which predominate as the causative agents and were resistant to commonly used cephalosporins and aminoglycosides, which are freely supplied in the government setups. The mode of death is probably due to endotoxin mediated multiple organ dysfunction. The death rate is also high because a higher proportion of babies are preterm and low birth weight, who lacked the inherent immunity to combat infection.

Signs and symptoms of sepsis are subtle and more difficult to recognize at early stages of the infection and ominous signs like shock, grunt, increasing abdominal girth with bilious 
vomiting and bleeding manifestations are late and uniformly fatal. Blood culture though considered gold standard, the culture positive rates are low. Sepsis screening parameters using CRP and haematological parameters are easily available, rapid, cost effective screening tests with good sensitivity and negative predictive value so that if any of the two screening tests are negative, the infection is unlikely. Neutropenia and thrombocytopenia have high specificity and are predictors of mortality. CRP is a good diagnostic and therapeutic tool. It has high sensitivity and a good negative predictive value so that the antibiotic can be started in culture negative and asymptomatic cases. This helps to reduce the delay in instituting antibiotic therapy.

Perinatal risk score involving intrapartum risk factors should be used for triage of high risk babies. Rapid diagnostic tests like CRP, WBC indices can be used as a screening means for early diagnosis and treatment of sepsis.

\section{References}

Anita Chandna, Nagaraj Rao M, Srinivas M, Shyamala S. Rapid diagnostic tests in, neonatal septicemia. Indian $\mathrm{J}$ Pediatr 1988; 55(6): 947-53.

Barbara L. Manroe, Arthur G. Weinberg, Charles R. Rosenfeld, Richard Browne. The neonatal blood count in health and disease. I. Reference values for neutrophilic cells. J Pediatr 1979 Jul; 95(1): 89-98

Bhakoo ON. Neonatal bacterial infections at Chandigarh: A decade of experience. Indian J Pediatr 1980; 47(388): 419-24.

CLSI. Performance Standards for Antimicrobial Susceptibility Testing; Twentieth Informational Supplement. CLSI document M100-S20. Wayne, PA: Clinical and Laboratory Standards Institute; 2010.
Collee GJ, Marimon BP, Frazer AG, Simmons A Mackie McCartney Practical Medical Microbiology. Collee JG, 14ed; 11:245-258, 4:151-177.

Elizabeth Mathai, Usha Christopher, Matthews Mathai, Atanu Kumar Jana, Dolly Rose, Staffan Bergstrom. Is CReactive Protein level useful in differentiating infected from noninfected neonates among those at risk of infection? Indian Pediatrics 2004; 41: 895-900.

Gerdes JS. Diagnosis and management of bacterial infection in neonate. Paediatric Clin N Am 2004; 51: 939-959.

Joseph W. St. Geme Jr, Dennis L. Murray, JoAnne Carter, Calvin J. Hobel, Rosemary D. Leake, Bascom F. Anthony et al., Perinatal bacterial infection after prolonged rupture of amniotic membranes: An analysis of risk and management. J Pediatr 1984 Apr; 104(4): 608-13.

Joshi SG, Ghole VS, Niphadkar KB. Neonatal Gram-Negative Bacteremia. Indian J Pediatr 2000; 67(1): 27-32.

Khatua SP, Das AK, Chatterjee BD, Khatua S, Ghose B, Saha A. Neonatal septicemia. Indian J Pediatr 1986; 53 (4): 509-14.

Madhu Sharma, Nidhi Goel, Uma Chaudhary, Ritu Aggarwal, Arora DR. Bacteraemia in Children. Indian J Pediatr 2002 Dec; 69(12): 1029-32.

National Neonatal Perinatal Database. Report 2002-03. Published by NNPD nodal centre, Department of Pediatrics, All India Institute of Medical Science, New Delhi.

Philip AG, and Mills PC. Use of C-reactive protein in minimizing antibiotic exposure. Experience with infants initially admitted to a well-baby nursery Pediatrics 2000; 106: E4. Prediction of Early Neonatal Infections. Indian Pediatrics 1974 Sept; 11(9): 597-600. 
Rekha Sriram: Correlation of blood culture results with the sepsis score and the sepsis screen in the diagnosis of neonatal septicemia. Int. Journal of Biological and Medical Research, 2011; 2(1): 360-8.

Rodwell RL, Anton LL, David IT. Early diagnosis of neonatal sepsis using a hematological scoring system. J Pediatrics 1987; 12; 761-67.

Ronald Misquith, Prakash Saldanha. Varadaraj Shenoy K, Sanjeev Rai B. The Use of Buffy Coat Smear for Diagnosis of Neonatal Septicaemia. Karnataka Pediatric Journal 2004 JanMar; 18(1): 9-13.

Roy I, Jain A, Kumar M, Agarwal SK. Bacteriology of Neonatal Septicaemia in a Tertiary care Hospital of Northern India. Indian Journal of Medical Microbiology 2002 Jul; 20(3): 156-9.

Sharmila Ghosh, Meenu Mittal, Geetha Jaganathan. Early Diagnosis of Neonatal Sepsis using a Hematological Scoring System. Ind J Med Sci 2001
Sept; 55(9): 495-500.

Stoll B J, Gordon T, Sheldon BK, et al., Late onset sepsis in very low birth weight neonates: A report from the NTCHD Neonatal Research Network. J Paediatrics 1996; 129:63-71.

Tallur SS, Kasturi AV, Shobha D. Nadgir, Krishna BVS. Clinico-bacteriologicai Study of Neonatal Septicemia in Hubli. Indian J Pediatr., 2000; 67(3): 169- 74.

Varsha, Usha Rusia, Meera Sikka, Faridi MMA, Nishi Madan. Validity of hematologic parameters in identification of early and late onset neonatal infection. Indian J Pathol Microbiol 2003; 46(4): 565-8.

Ved Parkash Takkar, Omkar Nath Bhakoo, Anil Narang. Scoring System for the Prediction of Early Neonatal Infections. Indian Pediatrics 1974 Sept; 11(9): 597600.

Yancey MK, Patric D, Paul K et al., Risk factors for neonatal sepsis. Obstetrics and Gynecology 1996; 87: 188-94.

\section{How to cite this article:}

Lazarus Monica, J. Seth Riti and Kinnare Amit, B. 2018. Role of Sepsis Screen Parameters in Early Diagnosis of Neonatal Septicemia. Int.J.Curr.Microbiol.App.Sci. 7(01): 2410-2419. doi: https://doi.org/10.20546/ijcmas.2018.701.290 